\title{
A matemática e a epidemia
}

\author{
Selma Helena de Jesus Nicola(D)
}

\begin{abstract}
Resumo
Para a dedução de uma Curva Epidêmica são utilizadas várias ferramentas matemáticas que podem ser exploradas no ensino. Este artigo tem como objetivo ser um material de apoio teórico para o professor responder perguntas de alunos sobre aspectos da epidemia e a Curva Epidêmica, destacando os tópicos de matemática utilizados.
\end{abstract}

Palavras-chave: Curva Epidêmica; limiar de uma epidemia; ensino; Modelo SIR.

\begin{abstract}
Many elementary mathematical tools are used to get an Epidemic Curve. This article aims to be a theoretical support for teachers to answer student questions about epidemic features and the Epidemic Curve pointing out some useful mathematical topics.
\end{abstract}

Keywords: Epidemic curve; epidemic threshold; teaching; SIR Model.

\section{Introdução}

Nos dias atuais estamos vivendo a crise de uma epidemia e ouvindo muito sobre o pico da curva epidêmica. Mostramos como chegar a uma representação dessa curva em um modelo matemático de uma doença com determinadas características de infecção, cura, mortalidade e condições sobre a população, que é dividida em indivíduos suscetíveis, infectados e removidos, como a Covid-19. Trata-se do bem conhecido Modelo SIR que é, na verdade, um sistema de equações diferenciais ordinárias aqui apresentado na linha do livro de Martin Braun [2], nossa referência básica, também utilizada para o Trabalho de Conclusão de Curso [3].

Para uma solução $(\mathrm{S}(\mathrm{t}), \mathrm{I}(\mathrm{t}), \mathrm{R}(\mathrm{t}))$ do Sistema SIR, a Curva Epidêmica é representada pelo gráfico da componente I(t), que descreve a evolução da classe de indivíduos infectados na população.

Entre os conhecimentos de matemática necessários, além da Álgebra Elementar, estão ferramentas básicas de Cálculo Diferencial e Integral e Equações Diferenciais Ordinárias, para os quais sugerimos [4] e [1]. Assim, podemos aproveitar a crise para motivar os alunos no estudo dessas disciplinas. Destacamos os seguintes tópicos: fatoração de polinômio, resolução de equação de segundo grau, função hiperbólica, limite, derivada, Regra da Cadeia, Teorema Fundamental do Cálculo, Série de Taylor, análise qualitativa de gráfico, integral, mudança de variável, modelagem, ponto de equilíbrio, órbita, plano de fase, equação de variáveis separáveis. 


\section{O Modelo}

Consideramos que uma população tenha sido afetada pelo aparecimento de um microrganismo que provoca uma doença contagiosa. Vamos dividi-la nas classes

(S) dos suscetíveis, que consiste daqueles indivíduos que não foram infectados mas estão sujeitos a contrair a doença,

(I) dos infectados, que consiste daqueles indivíduos que são capazes de transmitir a doença para outros,

(R) dos removidos, que consiste daqueles indivíduos que se recuperaram, estão se recuperando em isolamento ou foram a óbito.

Supomos as seguintes hipóteses:

- A população permanece num valor fixo N no intervalo de tempo considerado, isto é, desprezamos nascimentos, mortes por causas não relacionadas à doença em questão, imigração e emigração.

- A doença confere imunidade a todo indivíduo que foi infectado e tenha se recuperado.

- Um indivíduo da classe S que contrai a doença passa imediatamente para a classe I.

- A taxa de variação da população suscetível é proporcional ao produto do número de indivíduos da classe $\mathrm{S}$ e o número de indivíduos da classe $\mathrm{I}$, ou seja, é proporcional ao número de encontros de indivíduos dessas classes.

- Indivíduos são removidos a uma taxa proporcional ao tamanho da classe I.

As funções $\mathrm{S}(\mathrm{t}), \mathrm{I}(\mathrm{t}), \mathrm{R}(\mathrm{t})$ dadas pelo número de indivíduos no instante $\mathrm{t}$ das classes $\mathrm{S}, \mathrm{I}$ e $\mathrm{R}$, respectivamente, satisfazem então o sistema de equações diferenciais

$$
\begin{aligned}
& \frac{\mathrm{dS}}{\mathrm{dt}}=-\mathrm{rSI} \\
& \frac{\mathrm{dI}}{\mathrm{dt}}=\mathrm{rSI}-\gamma \mathrm{I} \\
& \frac{\mathrm{dR}}{\mathrm{dt}}=\gamma \mathrm{I},
\end{aligned}
$$

onde $\mathrm{r}$ e $\gamma$ são as constantes positivas de contágio e de remoção, respectivamente.

\section{Ocorrência ou não de epidemia}

Analisamos as soluções do Sistema (1) para saber como será a propagação da doença e, como as primeiras equações não dependem de R, concentramo-nos no sistema

$$
\begin{aligned}
& \frac{\mathrm{dS}}{\mathrm{dt}}=-\mathrm{rSI} \\
& \frac{\mathrm{dI}}{\mathrm{dt}}=\mathrm{rSI}-\gamma \mathrm{I} .
\end{aligned}
$$


Observamos que a função $R(t)$ pode ser obtida da terceira equação do Sistema (1) ou, como $\mathrm{S}(\mathrm{t})+\mathrm{I}(\mathrm{t})+\mathrm{R}(\mathrm{t})=\mathrm{N}$, temos $\mathrm{R}(\mathrm{t})=\mathrm{N}-\mathrm{S}(\mathrm{t})-\mathrm{I}(\mathrm{t})$.

Uma órbita do Sistema (2) é a curva $(\mathrm{S}(\mathrm{t}), \mathrm{I}(\mathrm{t}))$ descrita pela solução no chamado plano de fase SI. Uma das vantagens de considerar a órbita é a de ser frequentemente possível descrevê-la sem o conhecimento da correspondente solução. Os pontos de equilíbrio do Sistema (2) são as soluções constantes, ou seja, os pontos que anulam os segundos membros. Note que os pontos de equilíbrio do Sistema (2) são os pontos $(\mathrm{S}, 0)$ do eixo S. Além disso, o semieixo I positivo é uma órbita, pois a solução que passa por qualquer ponto $\left(0, \mathrm{I}_{0}\right), \mathrm{I}_{0}>0$, é $\left(0, \mathrm{I}_{0} \mathrm{e}^{-\gamma \mathrm{t}}\right),-\infty<\mathrm{t}<\infty$. Assim, como duas órbitas nunca se cruzam, todas as órbitas com condição inicial no primeiro quadrante permanecem nele. Esse fato revela que o sistema é coerente com a realidade, pois em nenhum instante a população de uma classe pode ser negativa.

De agora em diante supomos o instante inicial $t_{0}=0$ e que o número de indivíduos suscetíveis, infectados e removidos nesse instante sejam $\mathrm{S}_{0}, \mathrm{I}_{0}>0$ e $\mathrm{R}_{0}=0$, respectivamente. Ou seja, vamos considerar uma condição inicial $(\mathrm{S}(0), \mathrm{I}(0), \mathrm{R}(0))=\left(\mathrm{S}_{0}, \mathrm{I}_{0}, 0\right)$, portanto $\mathrm{S}(\mathrm{t}), \mathrm{I}(\mathrm{t})$ e $\mathrm{R}(\mathrm{t})$ positivos para $\mathrm{t}>0$. De acordo com (2), a componente $\mathrm{S}(\mathrm{t})$ é decrescente e, portanto, vamos nos ater a $\mathrm{S}<\mathrm{S}_{0}$.

Como estamos restritos ao quadrante $\mathrm{S}, \mathrm{I}>0$, podemos utilizar a regra da derivação da função inversa e a regra da cadeia para obter as órbitas do Sistema (2) como as curvas soluções da equação diferencial ordinária de primeira ordem

$$
\frac{\mathrm{dI}}{\mathrm{dS}}=\frac{\mathrm{dI}}{\mathrm{dt}} \frac{\mathrm{dt}}{\mathrm{dS}}=\frac{\mathrm{rSI}-\gamma \mathrm{I}}{-\mathrm{rSI}}=-1+\frac{\rho}{\mathrm{S}},
$$

onde definimos $\rho=\gamma / \mathrm{r}$.

Integrando a Equação (3) de $\mathrm{S}_{0}$ a $\mathrm{S}$ obtemos

$$
\mathrm{I}(\mathrm{S})=\mathrm{I}_{0}+\mathrm{S}_{0}-\mathrm{S}+\rho \ln \frac{\mathrm{S}}{\mathrm{S}_{0}} .
$$

A função $I(S)$ está definida para todo $S>0$, mas é solução da Equação (3) somente no intervalo em que $\mathrm{I}(\mathrm{S})>0$. Para analisar o comportamento das curvas (4), observamos que

- $\mathrm{I}(\mathrm{S})$ é decrescente se $\mathrm{dI} / \mathrm{dS}=-1+\rho / \mathrm{S}<0$, ou seja, $\mathrm{S}>\rho$,

- $\mathrm{I}(\mathrm{S})$ é crescente se $\mathrm{dI} / \mathrm{dS}=-1+\rho / \mathrm{S}>0$, ou seja, $\mathrm{S}<\rho$.

Portanto, $\mathrm{S}=\rho$ é o único ponto de máximo da função $\mathrm{I}(\mathrm{S})$, ou seja, de qualquer solução $\mathrm{I}(\mathrm{S})$ da Equação (3).

Como $\lim _{\mathrm{S} \rightarrow 0} \mathrm{I}(\mathrm{S})=-\infty$ e $\mathrm{I}(\rho) \geq \mathrm{I}\left(\mathrm{S}_{0}\right)=\mathrm{I}_{0}>0$, sendo $\mathrm{I}(\mathrm{S})$ contínua e crescente no intervalo $(0, \rho)$, segue do Teorema do Anulamento que existe um único ponto $\mathrm{S}_{\infty} \in(0, \rho]$ tal que $\mathrm{I}\left(\mathrm{S}_{\infty}\right)=0$.

$\mathrm{O}$ ponto $\left(\mathrm{S}_{\infty}, 0\right)$ é um ponto de equilíbrio do Sistema $(2)$, e, como as coordenadas $\mathrm{S}(\mathrm{t})$ e $\mathrm{I}(\mathrm{t})$ da órbita são decrescentes e limitadas para $\mathrm{S}<\rho$, então, $\lim _{\mathrm{t} \rightarrow \infty}(\mathrm{S}(\mathrm{t}), \mathrm{I}(\mathrm{t}))$ deve ser um ponto de equilíbrio. Logo, $\lim _{\mathrm{t} \rightarrow \infty}(\mathrm{S}(\mathrm{t}), \mathrm{I}(\mathrm{t}))=\left(\mathrm{S}_{\infty}, 0\right)$.

Portanto, as órbitas do Sistema (2) têm a forma que se indica na Figura 1, e, quando t varia de 0 até $\infty$, o ponto $(\mathrm{S}(\mathrm{t}), \mathrm{I}(\mathrm{t}))$ percorre a órbita movendo-se na direção de $\mathrm{S}$ decrescente. Assim, 
- se $\mathrm{S}_{0}<\rho, \mathrm{S}(\mathrm{t})$ decresce a $\mathrm{S}_{\infty}$ e $\mathrm{I}(\mathrm{t})$ decresce à extinção. Nesse caso não ocorre epidemia.

- se $\mathrm{S}_{0}>\rho, \mathrm{I}(\mathrm{t})$ cresce enquanto $\mathrm{S}(\mathrm{t})$ decresce para $\rho$ e atinge um valor máximo quando $\mathrm{S}=\rho$. A partir desse instante $\mathrm{I}(\mathrm{t})$ e $\mathrm{S}(\mathrm{t})$ decrescem com $\mathrm{I}(\mathrm{t})$ tendendo à extinção. Este é o caso em que ocorre uma epidemia.

Concluímos que ocorre uma epidemia se, e somente se, o número inicial $\mathrm{S}_{0}$ de indivíduos suscetíveis for maior que o número $\rho$. Seja qual for a condição inicial $\left(\mathrm{S}_{0}, \mathrm{I}_{0}\right)$, com $\mathrm{S}_{0}>\rho$, a órbita correspondente atinge um máximo para $\mathrm{S}=\rho$; portanto, $\rho$ é uma característica da enfermidade. $\mathrm{O}$ número $\rho$ é chamado limiar (threshold) da epidemia.

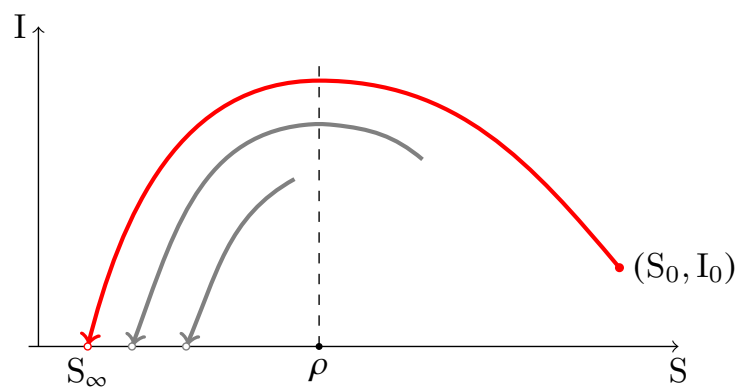

Figura 1: Órbita por $\left(\mathrm{S}_{0}, \mathrm{I}_{0}\right)$ no plano de fase SI.

Uma estratégia para controlar uma epidemia é aumentar o limiar $\rho$ aproximando-o do valor inicial $\mathrm{S}_{0}$ de indivíduos suscetíveis. Isso é possível aumentando $\gamma$ e ou diminuindo $\mathrm{r}$. Na prática, o aumento da constante $\gamma$ pode ser obtido melhorando as condições hospilares e investindo em pesquisas médicas. Para diminuir a constante $\mathrm{r}$ pode-se praticar o afastamento social e estimular condutas de proteção pessoal. Veremos mais adiante que a diminuição de r tem também a contribuição de diminuir o valor máximo $\mathrm{I}(\rho)$ de indivíduos infectados.

No caso de existir uma vacina, a estratégia seria diminuir o $\mathrm{S}_{0}$ visando tornar $\mathrm{S}_{0}<\rho$.

4. Os valores finais $S_{\infty}$ e $R_{\infty}$

$\mathrm{O}$ valor $\mathrm{I}_{\infty}$ foi excluído do título desta seção, pois já sabemos que $\mathrm{I}_{\infty}=\lim _{\mathrm{t} \rightarrow \infty} \mathrm{I}(\mathrm{t})=0$.

A função $\mathrm{S}(\mathrm{t})$ é decrescente, mas ao final de uma epidemia sempre restará uma quantidade $\mathrm{S}_{\infty}=$ $\lim _{t \rightarrow \infty} \mathrm{S}(\mathrm{t})$ de indivíduos suscetíveis. Tal fato torna provável ressurgimentos de epidemias da mesma enfermidade.

A função $R(t)$ é crescente, e portanto, $R_{\infty}$ é maior que $R(t)$ em qualquer momento da epidemia. Observamos que a quantidade total $R_{\infty}=\lim _{t \rightarrow \infty} R(t)$ de indivíduos recuperados é fornecida por $\mathrm{R}_{\infty}=\mathrm{I}_{0}+\mathrm{S}_{0}-\mathrm{S}_{\infty}$, e para obtê-la basta encontrar $\mathrm{S}_{\infty}$.

Fazendo $\mathrm{t} \rightarrow \infty$ em (4) temos $0=\mathrm{I}_{0}+\mathrm{S}_{0}-\mathrm{S}_{\infty}+\rho \ln \left(\mathrm{S}_{\infty} / \mathrm{S}_{0}\right)$, o que implica

$$
\ln \frac{\mathrm{S}_{\infty}}{\mathrm{S}_{0}}=\frac{1}{\rho}\left(\mathrm{S}_{\infty}-\mathrm{S}_{0}-\mathrm{I}_{0}\right) .
$$

Da primeira equação do Sistema (2) temos 


$$
\frac{\mathrm{dS}}{\mathrm{S}}=-\mathrm{rIdt} \Rightarrow \ln \frac{\mathrm{S}}{\mathrm{S}_{0}}=-\mathrm{rIt} \Rightarrow \mathrm{S}=\mathrm{S}_{0} \mathrm{e}^{-\mathrm{rIt}} \quad \text { e } \quad \mathrm{It}=-\frac{1}{\mathrm{r}} \ln \frac{\mathrm{S}}{\mathrm{S}_{0}} .
$$

Substituindo It em $\mathrm{S}=\mathrm{S}_{0} \mathrm{e}^{-\mathrm{rIt}}$ e fazendo $\mathrm{t} \rightarrow \infty$ obtemos $\mathrm{S}_{\infty}=\mathrm{S}_{0} \mathrm{e}^{\left(\mathrm{S}_{\infty}-\mathrm{S}_{0}-\mathrm{I}_{0}\right) / \rho}$, ou seja, $\mathrm{S}_{\infty}$ é raiz da equação

$$
\mathrm{S}=\mathrm{S}_{0} \mathrm{e}^{\left(\mathrm{S}-\mathrm{S}_{0}-\mathrm{I}_{0}\right) / \rho}
$$

Essa equação não pode ser resolvida algebricamente, mas afirmamos que ela possui duas raízes $\mathrm{S}_{\infty}$ e $S^{\prime}$. Buscar as soluções da Equação (5) é equivalente a encontrar as raízes da função

$$
f(S)=S-S_{0} e^{\left(S-S_{0}-I_{0}\right) / \rho} .
$$

Observando que $\mathrm{d}^{2} \mathrm{f} / \mathrm{dS}^{2}=-\left(\mathrm{S}_{0} / \rho^{2}\right) \mathrm{e}^{\left(\mathrm{S}-\mathrm{S}_{0}-\mathrm{I}_{0}\right) / \rho}$ é negativa, podemos garantir que o gráfico de $\mathrm{f}$ tem a concavidade voltada para baixo. Como $\lim _{S \rightarrow \pm \infty} \mathrm{f}(\mathrm{S})=-\infty$ a f tem um ponto de máximo e, sendo $\mathrm{f}\left(\mathrm{S}_{0}\right)=\mathrm{S}_{0}\left(1-\mathrm{e}^{-\mathrm{I}_{0} / \rho}\right)$ positivo, segue que f possui duas raízes, a primeira igual a $\mathrm{S}_{\infty}<\mathrm{S}_{0}$ e a segunda $\mathrm{S}^{\prime}$ que será desprezada por satisfazer $\mathrm{S}^{\prime}>\mathrm{S}_{0}$.

\section{A Curva Epidêmica}

Nesta seção obtemos as funções coordenadas $\mathrm{I}(\mathrm{t})$ e R(t). A Curva Epidêmica é o gráfico da função $\mathrm{I}(\mathrm{t})$. Note que, conhecidos $\mathrm{I}(\mathrm{t})$ e $\mathrm{R}(\mathrm{t})$, obtemos $\mathrm{S}(\mathrm{t})=\mathrm{N}-\mathrm{I}(\mathrm{t})-\mathrm{R}(\mathrm{t})$.

Enquanto está ocorrendo uma epidemia, conseguimos registrar o número de pessoas que procuram ajuda médica e o número de óbitos, ou seja, conseguimos registrar somente o número de novos indivíduos removidos. Sendo assim, podemos conhecer $\mathrm{dR} / \mathrm{dt}$.

Pelas regras da cadeia e da derivação da função inversa, $\mathrm{dS} / \mathrm{dR}=(\mathrm{dS} / \mathrm{dt})(\mathrm{dt} / \mathrm{dR})=(\mathrm{dS} / \mathrm{dt})(\mathrm{dR} / \mathrm{dt})^{-1}$ ou seja,

$$
\frac{\mathrm{dS}}{\mathrm{dR}}=\frac{-\mathrm{rSI}}{\gamma \mathrm{I}}=\frac{-\mathrm{S}}{\rho},
$$

e, resolvendo esta equação diferencial ordinária, obtemos

$$
\mathrm{S}(\mathrm{R})=\mathrm{S}_{0} \mathrm{e}^{-\mathrm{R} / \rho}
$$

Como

$$
\frac{\mathrm{dR}}{\mathrm{dt}}=\gamma \mathrm{I}=\gamma(\mathrm{N}-\mathrm{R}-\mathrm{S})
$$

temos

$$
\frac{\mathrm{dR}}{\mathrm{dt}}=\gamma\left(\mathrm{N}-\mathrm{R}-\mathrm{S}_{0} \mathrm{e}^{-\mathrm{R} / \rho}\right)
$$

A epidemia é em geral pequena em relação a N. Assim, S é sempre um número grande em comparação com R, e, como $\rho$ é um valor de $\mathrm{S}$, temos $\mathrm{R} / \rho$ muito pequeno. Logo, é possível truncar a Série de Taylor

$$
\mathrm{e}^{-\mathrm{R} / \rho}=1-\frac{\mathrm{R}}{\rho}+\frac{1}{2}\left(\frac{\mathrm{R}}{\rho}\right)^{2}+\cdots
$$

depois do terceiro termo. 
Com tal aproximação obtemos

$$
\frac{\mathrm{dR}}{\mathrm{dt}}=\gamma\left[\mathrm{N}-\mathrm{R}-\mathrm{S}_{0}\left(1-\frac{\mathrm{R}}{\rho}+\frac{1}{2}\left(\frac{\mathrm{R}}{\rho}\right)^{2}\right)\right]=\gamma\left[\mathrm{N}-\mathrm{S}_{0}+\left(\frac{\mathrm{S}_{0}}{\rho}-1\right) \mathrm{R}-\frac{\mathrm{S}_{0}}{2}\left(\frac{\mathrm{R}}{\rho}\right)^{2}\right],
$$

ou seja,

$$
\frac{\mathrm{dR}}{\mathrm{dt}}=\gamma\left[\left(\mathrm{N}-\mathrm{S}_{0}\right)+\left(\frac{\mathrm{S}_{0}}{\rho}-1\right) \mathrm{R}-\left(\frac{\mathrm{S}_{0}}{2 \rho^{2}}\right) \mathrm{R}^{2}\right]
$$

Definindo as variáveis

$$
\mathrm{A}=-\frac{\mathrm{S}_{0}}{2 \rho^{2}}, \quad \mathrm{~B}=\frac{\mathrm{S}_{0}}{\rho}-1, \quad \mathrm{C}=\mathrm{N}-\mathrm{S}_{0}
$$

e, substituindo em (8), obtemos a equação de variáveis separáveis

$$
\frac{\mathrm{dR}}{\mathrm{AR}^{2}+\mathrm{BR}+\mathrm{C}}=\gamma \mathrm{dt} \text {. }
$$

Observando que $\mathrm{B}^{2}-4 \mathrm{AC}>0$, pois $\mathrm{N}>\mathrm{S}_{0}$, podemos definir

$$
\alpha=\sqrt{\mathrm{B}^{2}-4 \mathrm{AC}}=\left[\left(\frac{\mathrm{S}_{0}}{\rho}-1\right)^{2}+\frac{2 \mathrm{~S}_{0}\left(\mathrm{~N}-\mathrm{S}_{0}\right)}{\rho^{2}}\right]^{1 / 2}
$$

e a equação do segundo grau $\mathrm{AR}^{2}+\mathrm{BR}+\mathrm{C}=0$, possui, então as raízes

$$
\mathrm{R}=\frac{-\mathrm{B} \pm \sqrt{\mathrm{B}^{2}-4 \mathrm{AC}}}{2 \mathrm{~A}}=\frac{-\mathrm{B} \pm \alpha}{2 \mathrm{~A}}=\rho-\frac{\rho^{2}}{\mathrm{~S}_{0}} \pm \frac{\rho^{2}}{\mathrm{~S}_{0}} \alpha
$$

e, definindo as variáveis

$$
\mathrm{D}=\rho-\frac{\rho^{2}}{\mathrm{~S}_{0}} \quad \text { e } \quad \mathrm{E}=\frac{\rho^{2}}{\mathrm{~S}_{0}}
$$

tornam-se

$$
\mathrm{R}=\mathrm{D} \pm \mathrm{E} \alpha
$$

Podemos fatorar $\mathrm{AR}^{2}+\mathrm{BR}+\mathrm{C}$ da seguinte maneira

$$
\mathrm{A}[\mathrm{R}-(\mathrm{D}+\mathrm{E} \alpha)][\mathrm{R}-(\mathrm{D}-\mathrm{E} \alpha)]=\mathrm{A}[(\mathrm{R}-\mathrm{D})+\mathrm{E} \alpha][(\mathrm{R}-\mathrm{D})-\mathrm{E} \alpha)]=\mathrm{A}\left[(\mathrm{R}-\mathrm{D})^{2}-\mathrm{E}^{2} \alpha^{2}\right] .
$$

Assim,

$$
\int \frac{\mathrm{dR}}{\mathrm{AR}^{2}+\mathrm{BR}+\mathrm{C}}=\frac{1}{\mathrm{~A}} \int \frac{\mathrm{dR}}{(\mathrm{R}-\mathrm{D})^{2}-\mathrm{E}^{2} \alpha^{2}} .
$$

Fazendo a mudança de variável $\mathrm{x}=\mathrm{R}-\mathrm{D}$, e definindo $\mathrm{a}^{2}=\mathrm{E}^{2} \alpha^{2}$, temos

$$
\frac{1}{\mathrm{~A}} \int \frac{\mathrm{dR}}{(\mathrm{R}-\mathrm{D})^{2}-\mathrm{E}^{2} \alpha^{2}}=\frac{1}{\mathrm{~A}} \int \frac{\mathrm{dx}}{\mathrm{x}^{2}-\mathrm{a}^{2}},
$$

e, com uma nova mudança de variável $\mathrm{x}=\mathrm{a}$ tanh $\mathrm{u}$, temos

$$
\frac{1}{\mathrm{~A}} \int \frac{\mathrm{dx}}{\mathrm{x}^{2}-\mathrm{a}^{2}}=-\frac{1}{\mathrm{~A}} \int \frac{\mathrm{asech}^{2} \mathrm{u}}{\mathrm{a}^{2} \operatorname{sech}^{2} \mathrm{u}} \mathrm{du}=-\frac{1}{\mathrm{Aa}} \int \mathrm{d} u=-\frac{1}{\mathrm{Aa}} \mathrm{u}+\mathrm{K}=-\frac{1}{\mathrm{Aa}} \operatorname{arctanh} \frac{\mathrm{x}}{\mathrm{a}}+\mathrm{K},
$$


onde K é uma constante. Nas variáveis R, D, E temos

$$
\frac{1}{\mathrm{~A}} \int \frac{\mathrm{dR}}{(\mathrm{R}-\mathrm{D})^{2}-\mathrm{E}^{2} \alpha^{2}}=-\frac{1}{\mathrm{AE} \alpha} \operatorname{arctanh} \frac{\mathrm{R}-\mathrm{D}}{\mathrm{E} \alpha}+\mathrm{K}
$$

e, como

$$
\mathrm{R}-\mathrm{D}=\mathrm{R}-\left(\rho-\frac{\rho^{2}}{\mathrm{~S}_{0}}\right), \quad \mathrm{E} \alpha=\frac{\rho^{2}}{\mathrm{~S}_{0}} \alpha, \quad \mathrm{AE} \alpha=-\frac{\alpha}{2},
$$

temos

$$
-\frac{1}{\mathrm{AE} \alpha} \operatorname{arctanh} \frac{\mathrm{R}-\mathrm{D}}{\mathrm{E} \alpha}+\mathrm{K}=\frac{2}{\alpha} \operatorname{arctanh}\left\{\frac{1}{\alpha}\left[\frac{\mathrm{S}_{0}}{\rho^{2}} \mathrm{R}-\left(\frac{\mathrm{S}_{0}}{\rho}-1\right)\right]\right\}+\mathrm{K} .
$$

Voltando à Equação (9), temos

$$
\frac{2}{\alpha} \operatorname{arctanh}\left\{\frac{1}{\alpha}\left[\frac{\mathrm{S}_{0}}{\rho^{2}} \mathrm{R}-\left(\frac{\mathrm{S}_{0}}{\rho}-1\right)\right]\right\}+\mathrm{K}=\gamma \mathrm{t} .
$$

Observamos que para $t=0$ temos $R=0 \mathrm{e}$

$$
\mathrm{K}=\frac{2}{\alpha} \operatorname{arctanh}\left[\frac{1}{\alpha}\left(\frac{\mathrm{S}_{0}}{\rho}-1\right)\right] .
$$

Então,

$$
\operatorname{arctanh}\left\{\frac{1}{\alpha}\left[\frac{\mathrm{S}_{0}}{\rho^{2}} \mathrm{R}-\left(\frac{\mathrm{S}_{0}}{\rho}-1\right)\right]\right\}=\frac{1}{2} \alpha \gamma \mathrm{t}-\operatorname{arctanh}\left[\frac{1}{\alpha}\left(\frac{\mathrm{S}_{0}}{\rho}-1\right)\right],
$$

ou seja,

$$
\frac{1}{\alpha}\left[\frac{\mathrm{S}_{0}}{\rho^{2}} \mathrm{R}-\left(\frac{\mathrm{S}_{0}}{\rho}-1\right)\right]=\tanh \left(\frac{1}{2} \alpha \gamma \mathrm{t}-\phi\right),
$$

onde

$$
\phi=\operatorname{arctanh}\left[\frac{1}{\alpha}\left(\frac{\mathrm{S}_{0}}{\rho}-1\right)\right]
$$

Logo,

$$
\mathrm{R}(\mathrm{t})=\frac{\rho^{2}}{\mathrm{~S}_{0}}\left[\frac{\mathrm{S}_{0}}{\rho}-1+\alpha \tanh \left(\frac{1}{2} \alpha \gamma \mathrm{t}-\phi\right)\right] .
$$

Derivando $\mathrm{R}$ em relação a t na expressão (13), obtemos

$$
\frac{\mathrm{dR}}{\mathrm{dt}}=\frac{\gamma \alpha^{2} \rho^{2}}{2 \mathrm{~S}_{0}} \operatorname{sech}^{2}\left(\frac{1}{2} \alpha \gamma \mathrm{t}-\phi\right) .
$$

Como $\mathrm{dR} / \mathrm{dt}=\gamma \mathrm{I}$ temos finalmente

$$
\mathrm{I}(\mathrm{t})=\frac{\alpha^{2} \rho^{2}}{2 \mathrm{~S}_{0}} \operatorname{sech}^{2}\left(\frac{1}{2} \alpha \gamma \mathrm{t}-\phi\right) .
$$

Essa função tem um único ponto crítico $\overline{\mathrm{t}}=2 \phi / \alpha \gamma$, que obtemos resolvendo em $\mathrm{t}$ a equação $\mathrm{dI} / \mathrm{dt}=0$ e, como $\left(\mathrm{d}^{2} \mathrm{I} / \mathrm{dt}^{2}\right)(\overline{\mathrm{t}})<0$, temos que $\overline{\mathrm{t}}$ é ponto de máximo, sendo $\mathrm{I}(\overline{\mathrm{t}})=\alpha^{2} \rho^{2} / 2 \mathrm{~S}_{0}$ o valor máximo. Como a função secante hiperbólica é par, a função $\mathrm{I}(\mathrm{t})$ é simétrica em relação à reta $\mathrm{t}=\overline{\mathrm{t}}$ e tem dois pontos de inflexão também simétricos em relação a essa, os quais obtemos resolvendo em $t$ a equação $\mathrm{d}^{2} \mathrm{I} / \mathrm{dt}^{2}=0$. Como $\mathrm{I}(\mathrm{t})>0$, para todo $\mathrm{t}$, e $\lim _{\mathrm{t} \rightarrow \pm \infty} \mathrm{I}(\mathrm{t})=0$, o gráfico da função $\mathrm{I}(\mathrm{t})$ 


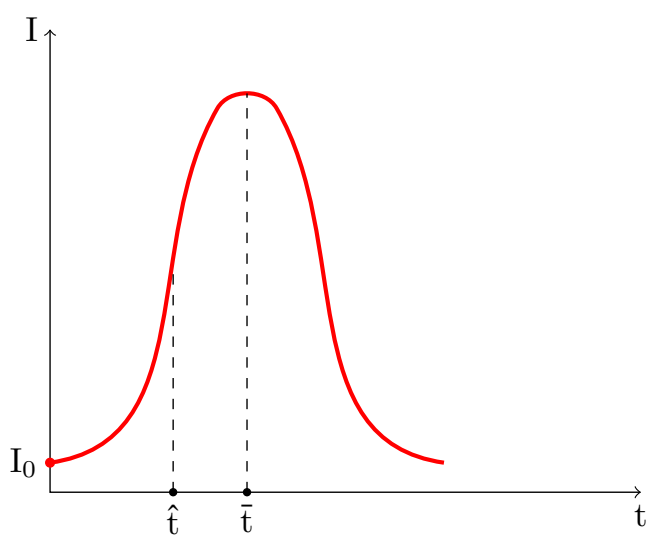

Figura 2: A Curva Epidêmica.

define uma curva simétrica com forma de sino conhecido como Curva Epidêmica da enfermidade; veja Figura 2.

Tal curva confirma a observação comum de que, em muitas epidemias reais, o número de novos casos reportados cada dia aumenta até alcançar o valor máximo $\mathrm{I}(\overline{\mathrm{t}})$ no instante $\overline{\mathrm{t}}$.

De acordo com (10) temos

$$
\alpha=\frac{1}{\rho} \sqrt{\left(\mathrm{S}_{0}-\rho\right)^{2}+2 \mathrm{~S}_{0}\left(\mathrm{~N}-\mathrm{S}_{0}\right)^{2}}
$$

e, portanto, o valor máximo $\mathrm{I}(\overline{\mathrm{t}})$, o chamado "pico da epidemia", satisfaz

$$
\mathrm{I}(\overline{\mathrm{t}})=\frac{\alpha^{2} \rho^{2}}{2 \mathrm{~S}_{0}}=\frac{1}{2 \mathrm{~S}_{0}}\left[\left(\mathrm{~S}_{0}-\rho\right)^{2}+2 \mathrm{~S}_{0}\left(\mathrm{~N}-\mathrm{S}_{0}\right)^{2}\right]
$$

no instante

$$
\overline{\mathrm{t}}=\frac{2 \phi}{\alpha \gamma}=\frac{2 \phi}{\mathrm{r} \sqrt{\left(\mathrm{S}_{0}-\rho\right)^{2}+2 \mathrm{~S}_{0}\left(\mathrm{~N}-\mathrm{S}_{0}\right)^{2}}} .
$$

Como, para que haja epidemia, é necessário $\mathrm{S}_{0}>\rho$, e, lembrando que $\rho=\gamma / \mathrm{r}$, se diminuímos a constante de contágio r, aumentamos $\rho$, e, portanto, de acordo com (16) e (17), diminuímos o valor do pico $\mathrm{I}(\overline{\mathrm{t}})$ e retardamos o instante $\overline{\mathrm{t}}$.

Analisando a Curva Epidêmica podemos entender o porquê de as autoridades de saúde falarem em "virada do jogo" antes do pico da epidemia. Isso se justifica porque a Curva Epidêmica começa com um crescimento acentuado e o gráfico tendo a convidade voltada para cima (a derivada é crescente). Se, porém, em um instante $\hat{t}$ anterior ao instante $\bar{t}$ do pico há uma inflexão, a concavidade volta-se para baixo (a derivada passa a decrescer). Por um lapso de tempo a epidemia ainda cresce, mas o crescimento começa a diminuir. A partir de $\hat{t}$, o instante da "virada", a epidemia tende de modo sustentável à extinção, passando a decrescer somente depois de $\overline{\mathrm{t}}$.

\section{Comentários Finais}

A utilização de modelos matemáticos tem sido uma ferramenta importante para o entendimento de uma epidemia. O conhecimento do Modelo SIR é importante para o sistema de saúde no 
planejamento do combate a uma epidemia, pois ele fornece a evolução da população de indivíduos infectados, a qual é, na verdade, a evolução da epidemia.

O contágio da Covid-19 tem um grau de nocividade maior do que as enfermidades mais conhecidas cujas epidemias são descritas pelo Modelo SIR. Um indivíduo infectado, por onde passa, vai deixando um rastro de focos de infecção que se desvanece em um tempo considerável. Assim, um indivíduo suscetível pode ser infectado por ele sem nunca tê-lo encontrado. Ou seja, a Covid-19 é mais contagiosa mas isso não inviabiliza o Modelo SIR para descrevê-la, basta tomar uma constante r maior.

A Curva Epidêmica real não tem as simetrias da forma de um sino, mas é uma deformação da curva estudada aqui. No mesmo sentido o limiar é apenas uma estimativa, mas é um parâmetro extremamente importante para o conhecimento da epidemia, pois a partir dele podemos estimar o valor e o instante do pico. É preciso entender que um modelo matemático é sempre uma aproximação da realidade, uma simplificação. Se não fosse assim, ele seria um retrato fiel, em geral tão complexo quanto a própria realidade.

Finalmente, na inexistência de vacina e considerando as dificuldades naturais de implementar rapidamente um tratamento clínico, resta uma única estratégia eficaz de combater uma epidemia. Trata-se de diminuir o contágio pelo distanciamento social e a difusão da proteção pessoal.

\section{Agradecimentos}

A autora agradece a P.Z. Táboas por algumas profícuas discussões.

\section{Referências}

[1] Boyce, W.E.; Diprima, R.C. Equações Diferenciais Elementares e Problemas de Valores de Contorno, 7a edição, LTC - Livros Técnicos e Científicos Editora, Rio de Janeiro, RJ, 2002.

[2] Braun, M. Differential Equations and their Applications, $4 \underline{a}$ edição, Springer, New York, NY, USA, 1993.

[3] Rodrigues, J.V.S. Um Modelo Matemático em Epidemiologia, Trabalho de Conclusão de Curso, Departamento de Matemática, UFSCar - Universidade Federal de São Carlos, São Carlos, SP, 2019.

[4] Táboas, P.Z. Cálculo em uma Variável Real, Edusp - Editora da Universidade de São Paulo, São Paulo, SP, 2008.

Selma Helena de Jesus Nicola Universidade Federal de São Carlos <selma.nicola@ufscar.br>

Recebido: 21/04/2020

Publicado: 02/07/2020 